\title{
Rapid diagnosis of schistosomiasis in Yemen using a simple questionnaire and urine reagent strips
}

H.K. Bassiouny, ${ }^{1}$ A.A. Hasab, ${ }^{2}$ N.A. El-Nimr, ${ }^{2}$ L.A. Al-Shibani ${ }^{3}$ and A.A. Al- Waleedi ${ }^{4}$

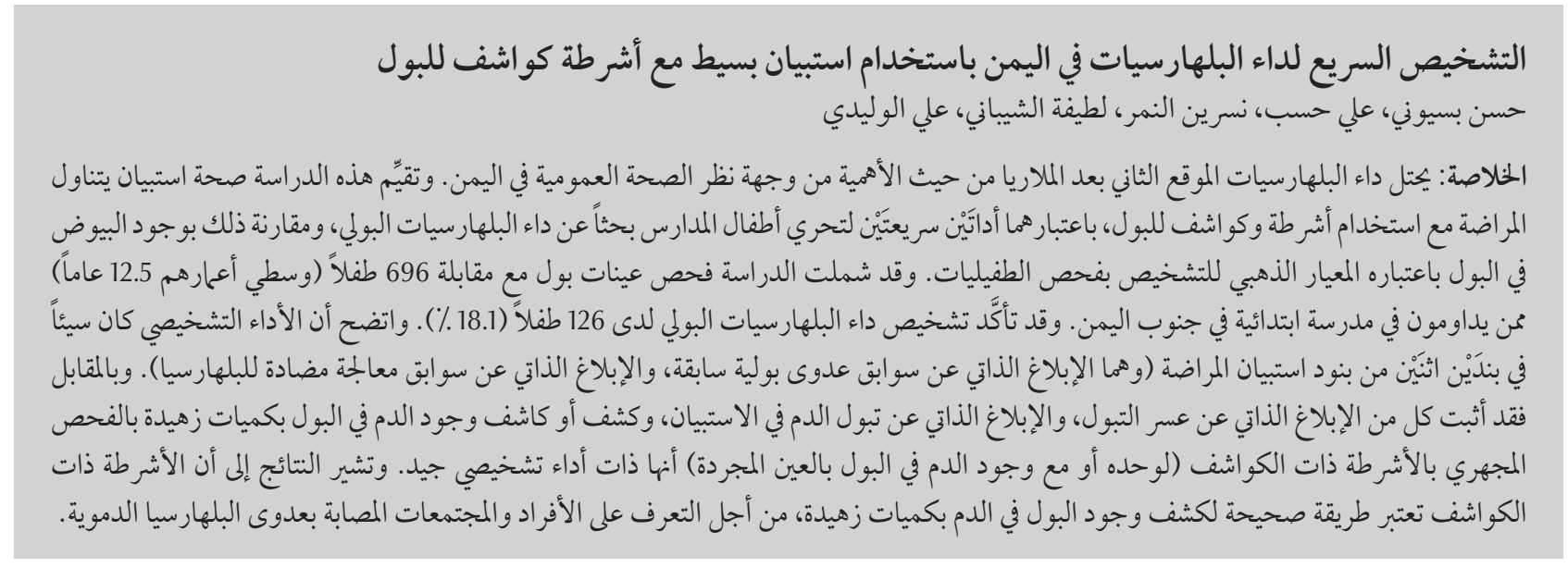

ABSTRACT Schistosomiasis ranks second to malaria in terms of socioeconomic and public health importance in Yemen. This study assessed the validity of a morbidity questionnaire and urine reagent strips as a rapid tool for screening schoolchildren for urinary schistosomiasis as compared with the presence of eggs in urine as the goldstandard parasitological diagnosis. The study examined urine samples and interviewed 696 children (mean age 12.5 years) attending a primary-preparatory school in south Yemen. Urinary schistosomiasis was confirmed in 126 (18.1\%) children. Diagnostic performance was poor for 2 items in the morbidity questionnaire (self-reported history of previous infection and self-reported history of antischistosomal treatment). However, self-reported dysuria, selfreported haematuria in the questionnaire and microhaematuria by reagent strips (alone or with macrohaematuria) revealed good diagnostic performance. The results indicated that reagent strips are a valid method for detection of microhaematuria for identifying individuals and communities infected with Schistosoma haematobium.

Diagnostic rapide de la schistosomiase au Yémen à l'aide d'un questionnaire simple et de bandelettes urinaires réactives

RÉSUMÉ La schistosomiase vient en deuxième place après le paludisme en termes de poids socioéconomique et de problème de santé publique au Yémen. La présente étude a évalué la validité d'un questionnaire sur la morbidité ainsi que des bandelettes urinaires réactives comme outils rapides de dépistage de la schistosomiase urinaire chez des écoliers par rapport à la recherche d'œufs dans les urines en tant que méthode diagnostique parasitologique de référence. Au cours de l'étude, les échantillons d'urine de 696 enfants fréquentant une école primaire ou préparatoire dans le sud du Yémen ont été examinés, puis les enfants ont été interrogés (âge moyen 12,5 ans). Une schistosomiase urinaire a été confirmée chez 126 enfants (18,1\%). La performance du diagnostic était médiocre pour deux items du questionnaire sur la morbidité (antécédents autodéclarés d'une infection antérieure et d'un traitement contre la schistosomiase). Toutefois, une dysurie autodéclarée, une hématurie autodéclarée dans le questionnaire et une microhématurie par bandelettes urinaires réactives (seule ou associée à une macrohématurie) ont fait ressortir une bonne performance diagnostique. Les résultats ont indiqué que les bandelettes urinaires réactives étaient une méthode valable pour le dépistage de la microhématurie permettant d'identifier les personnes et les communautés infestées par Schistosoma haematobium. 


\section{Introduction}

Schistosomiasis is a major public health problem in Yemen, second in importance only to malaria. It is estimated that 3 million people are infected and 600000 suffer clinical morbidity [1]. The disease is found in various areas mainly in Hajah, Ibb, Sana'a, Sada'a,Abyan and Taiz governorates. The recorded prevalence of urinary schistosomiasis in 2009 was $21.4 \%$ among schoolchildren in Abyan and Taiz governorates [2].

There is a need for updated information at governorate level in Yemen in order to map the disease geographically and to facilitate effective monitoring of the sustainability of prevention and control programmes at local level. A reliable and simple, rapid diagnostic method is needed to assess the extent of the disease burden, the communities at risk and the factors associated with the risk of infection and morbidity.

The aim of this study was to evaluate the utility of a simple morbidity questionnaire coupled with urine reagent strip (dipstick) tests for haematuria as a rapid screening tool for urinary schistosomiasis, compared with the gold-standard parasitological diagnosis, in one of the most endemic areas of Schistosoma haematobium infection in Yemen.

\section{Methods}

\section{Study area}

The study was carried out in Battis village, Abyan Governorate, in the south of Yemen. The selection of the area was based on reports from the central hospital, clinics and health centres of Abyan Governorate, where cases of urinary schistosomiasis had been reported. The village has 1 primary-preparatory mixed school located in the centre of the village. Three water ponds are mainly used for irrigation and domestic use due to inadequate water supplies. People, particularly children, visit these for swimming especially during the summer.

\section{Study population}

Prior to the commencement of the research ethical approval was sought from the ethics review board of the High Institute of Public Health, Alexandria University. The aims and procedures for data collection were explained to parents and community leaders including the school committees of Battis village. Oral consent was obtained from parents and consent was subsequently obtained from the children. The data collection period was from November 2010 to the end of June 2011.

A cross-sectional approach was used. All children of the 5th and 6th grades of primary education and 1 st and 2nd grades of preparatory education were eligible for the study. The overall sample size was 696 children (305 males and 391 females). The children were aged $10-16$ years, with a mean age of 12.5 (standard deviation 1.05) years.

\section{Design and use of the questionnaire}

A simple questionnaire was designed based on the key indicators of schistosomiasis. Each child was asked to respond (yes/no) to 4 questions about: presence of pain while urinating (in the last 2 weeks), presence of blood in urine (in the last 2 weeks), a history of previous infection with urinary schistosomiasis, and a history of antischistosomal treatment (in the 1 month prior to the study). At the preparatory level children filled the written questionnaire themselves, while at the primary level the children were assisted by the researchers and schoolteachers.

\section{Urine sample collection and examination}

Children were given dry clean plastic bottles to bring urine specimens. The containers were labelled with the same code number of the individual's questionnaire. The collection of terminal urine samples was carried out after short physical exercise between 10.00 and 14.00 hours, since this approach ensured that the eggs of S. haematobium were more likely to be passed [3].

Macroscopic examination was done for detection of visible haematuria (macrohaematuria) by naked eye examination and detection of microhaematuria by chemical reagent strips (UroColor 9, Standard Diagnostics).

The gold standard was microscopic examination of urine samples for detection and counting the number of $S$. haematobium eggs per $10 \mathrm{~mL}$ urine, which was done using the carbol-fuchsin centrifugal sedimentation technique [4].

\section{Data analysis}

Correlation of the questionnaire and reagent strip results with the gold-standard parasitological data was done using diagnostic accuracy tests [5]. Sensitivity, specificity, positive and negative predictive values (PPV and NPV), likelihood ratios for positive results $(\mathrm{LR}+)$ and receiver operating characteristics curve (ROC curve) and the area under the curve (AUC) were calculated. The chisquared test was used to compare the predictors of urinary schistosomiasis in relation to the prevalence and intensity of infection. $P<0.05$ was considered significant.

\section{Results}

A total of 696 children were interviewed and their urine examined. Based on microscopic examination of urine samples 126 children were found to be infected with urinary schistosomiasis (18.1\%).

\section{Predictors of urinary schistosomiasis}

Table 1 shows the responses to the morbidity questionnaire according to the prevalence of S. haematobium infection by microscopic examination. Of 


\begin{tabular}{|c|c|c|c|c|c|c|c|c|}
\hline \multirow{3}{*}{$\begin{array}{l}\text { Predictors of urinary } \\
\text { schistosomiasis }\end{array}$} & \multicolumn{4}{|c|}{ S. haematobium by microscopic examination } & \multicolumn{2}{|c|}{ Total $(n=696)$} & \multicolumn{2}{|c|}{ Statistics } \\
\hline & \multicolumn{2}{|c|}{$\begin{array}{l}\text { Positive } \\
(n=126)\end{array}$} & \multicolumn{2}{|c|}{$\begin{array}{l}\text { Negative } \\
(n=570)\end{array}$} & \multirow[b]{2}{*}{ No. } & \multirow[b]{2}{*}{$\%$} & \multirow[b]{2}{*}{$x^{2}$} & \multirow[b]{2}{*}{$P$-value } \\
\hline & No. & $\%$ & No. & $\%$ & & & & \\
\hline \multicolumn{9}{|l|}{ Questionnaire results } \\
\hline \multicolumn{9}{|l|}{ Self-reported dysuria } \\
\hline Yes & 99 & 78.6 & 108 & 18.9 & 207 & 29.7 & 175.6 & $<0.05$ \\
\hline No & 27 & 21.4 & 462 & 81.1 & 489 & 70.3 & & \\
\hline \multicolumn{9}{|c|}{ Self-reported haematuria } \\
\hline Yes & 58 & 46.0 & 18 & 3.2 & 76 & 10.9 & 195.0 & $<0.05$ \\
\hline No & 68 & 54.0 & 552 & 96.8 & 620 & 89.1 & & \\
\hline \multicolumn{9}{|c|}{ History of previous infection } \\
\hline Yes & 34 & 27.0 & 56 & 9.8 & 90 & 12.9 & 26.95 & $<0.05$ \\
\hline No & 92 & 73.0 & 514 & 90.2 & 606 & 87.1 & & \\
\hline \multicolumn{9}{|c|}{$\begin{array}{l}\text { History of antischistosomal } \\
\text { treatment }\end{array}$} \\
\hline Yes & 27 & 21.4 & 127 & 22.3 & 154 & 22.1 & 0.043 & NS \\
\hline No & 99 & 78.6 & 443 & 77.7 & 542 & 77.9 & & \\
\hline \multicolumn{9}{|l|}{ Dipstick/visual results } \\
\hline \multicolumn{9}{|l|}{ Microhaematuria only ${ }^{a}$} \\
\hline Positive & 78 & 61.9 & 34 & 6.0 & 112 & 16.1 & 235.0 & $<0.05$ \\
\hline Negative & 48 & 38.1 & 536 & 94.0 & 584 & 83.9 & & \\
\hline \multicolumn{9}{|l|}{$\begin{array}{l}\text { Both micro- + } \\
\text { macrohaematuria }^{b}\end{array}$} \\
\hline Positive & 39 & 31.0 & 0 & 0.0 & 39 & 5.6 & 181.1 & $<0.05$ \\
\hline Negative & 87 & 69.0 & 570 & 100.0 & 657 & 94.4 & & \\
\hline \multicolumn{9}{|l|}{ All microhaematuria } \\
\hline Positive & 117 & 92.9 & 34 & 6.0 & 151 & 21.7 & 417 & $<0.05$ \\
\hline Negative & 9 & 7.1 & 536 & 94.0 & 545 & 78.3 & & \\
\hline
\end{tabular}

aPostive by dipstick only; ${ }^{b}$ Postive by dipstick + visual inspection.

126 cases positive for $S$. haematobium infection $78.6 \%$ had self-reported dysuria compared with only $18.9 \%$ of the 570 children who were not infected. Haematuria and a history of previous infection were also reported by more of the infected than uninfected children ( $46.0 \%$ versus $3.2 \%$ and $27.0 \%$ versus $9.8 \%$ respectively). History of antischistosomal treatment was similar in infected and uninfected children $(21.4 \%$ versus $22.3 \%$ ).

Table 1 also shows the results of urine reagent strip testing and visual inspection for haematuria. Dipstick testing showed $61.9 \%$ of infected cases had microhaematuria compared with $6.0 \%$ of uninfected children, while $31.0 \%$ of cases had concomitant haematuria (i.e. both microhaematuria by dipstick plus macrohaematuria on visual inspection) versus none of the uninfected children. Thus there were 117 (92.9\%) children with either microhaematuria alone or microhaematuria plus macrohaematuria compared with only $6.0 \%$ who were not infected.

Table 2 shows the responses to the morbidity questionnaire and the results of dipstick testing and visual inspection of the urine samples according to the intensity of S. haematobiun infection. In general, the most of the infected children $(108 / 126,85.7 \%)$ had a low intensity of infection (1-49 eggs/ $10 \mathrm{~mL}$ urine). Infected cases with self-reported dysuria, self-reported haematuria, history of previous infection and history of antischistosomal treatment were of high intensity of infection (83.3\%, $72.2 \%, 27.8 \%$ and $33.3 \%$ respectively). However, there was no significant difference between those of high and low intensity of infection except with those of self-reported haematuria $\left(\chi^{2}=5.798\right.$, $P<0.05)$. Dipstick testing and visual inspection of urine samples showed $100 \%$ of cases who had both micro- plus macrohaematuria and all microhaematuria cases were of high intensity of infection, while all infected cases that 


\begin{tabular}{|c|c|c|c|c|c|c|c|c|}
\hline \multirow{3}{*}{$\begin{array}{l}\text { Predictors of urinary } \\
\text { schistosomiasis }\end{array}$} & \multicolumn{4}{|c|}{ S. haematobium by microscopic examination } & \multicolumn{2}{|c|}{ Total infected $(n=126)$} & \multicolumn{2}{|c|}{ Statistics } \\
\hline & \multicolumn{2}{|c|}{$\begin{array}{l}\text { High intensity } \\
\qquad(n=18)\end{array}$} & \multicolumn{2}{|c|}{$\begin{array}{l}\text { Low intensity } \\
\qquad(n=108)\end{array}$} & \multirow[b]{2}{*}{ No. } & \multirow[b]{2}{*}{$\%$} & \multirow[b]{2}{*}{$x^{2}$} & \multirow[b]{2}{*}{$P$-value } \\
\hline & No. & $\%$ & No. & $\%$ & & & & \\
\hline \multicolumn{9}{|l|}{ Questionnaire results } \\
\hline \multicolumn{9}{|l|}{ Self-reported dysuria } \\
\hline Yes & 15 & 83.3 & 84 & 77.8 & 99 & 78.6 & 0.283 & NS \\
\hline No & 3 & 16.7 & 24 & 22.2 & 27 & 21.4 & & \\
\hline \multicolumn{9}{|c|}{ Self-reported haematuria } \\
\hline Yes & 13 & 72.2 & 45 & 41.7 & 58 & 46.0 & 5.798 & $<0.05$ \\
\hline No & 5 & 27.8 & 63 & 58.3 & 68 & 54.0 & & \\
\hline \multicolumn{9}{|c|}{ History of previous infection } \\
\hline Yes & 5 & 27.8 & 29 & 26.9 & 34 & 27.0 & 0.007 & NS \\
\hline No & 13 & 72.2 & 79 & 73.1 & 92 & 73.0 & & \\
\hline \multicolumn{9}{|c|}{$\begin{array}{l}\text { History of antischistosomal } \\
\text { treatment }\end{array}$} \\
\hline Yes & 6 & 33.3 & 21 & 19.4 & 27 & 21.4 & 1.754 & NS \\
\hline No & 12 & 66.7 & 87 & 80.6 & 99 & 78.6 & & \\
\hline \multicolumn{9}{|l|}{ Dipstick/visual results } \\
\hline \multicolumn{9}{|l|}{ Microhaematuria only ${ }^{a}$} \\
\hline Positive & 0 & 0.0 & 78 & 72.2 & 78 & 61.9 & 31.13 & $<0.05$ \\
\hline Negative & 18 & 100.0 & 30 & 27.8 & 48 & 38.1 & & \\
\hline \multicolumn{9}{|l|}{$\begin{array}{l}\text { Both micro- + } \\
\text { macrohaematuria }^{b}\end{array}$} \\
\hline Positive & 18 & 100.0 & 21 & 19.4 & 39 & 31.0 & 43.15 & $<0.05$ \\
\hline Negative & 0 & 0.0 & 87 & 80.6 & 87 & 69.0 & & \\
\hline \multicolumn{9}{|l|}{ All microhaematuria } \\
\hline Positive & 18 & 100.0 & 99 & 91.7 & 117 & 92.9 & 0.603 & NS \\
\hline Negative & 0 & 0.0 & 9 & 8.3 & 9 & 7.1 & & \\
\hline
\end{tabular}

${ }^{a}$ High intensity $>50$ eggs $/ 10 \mathrm{~mL}$ urine; ${ }^{b}$ Low intensity $1-49$ eggs $/ 10 \mathrm{~mL}$ urine. NS = not significant.

were negative for microhaematuria only by dipstick had high intensity of infection by microscopic examination.

Table 3 shows the distribution of schoolchildren with self-reported haematuria by age and sex. Out of 76 selfreported haematuria 47 (61.8\%) were males and 29 (38.2\%) were females. Almost 52\% of females were within the age group 13-16 years.

\section{Diagnostic performance}

Table 4 shows the diagnostic performance of predictors as screening tools for urinary schistosomiasis among schoolchildren. The diagnostic performance of self-reported dysuria and self-reported haematuria had high to moderate sensitivity $(78.6 \%$ and $46.0 \%$ respectively) with high specificity ( $81.1 \%$ and $96.8 \%$ respectively). These data resulted in moderate to high PPV (47.8\% and 76.3\% respectively) and high NPV (94.5\% and $89.0 \%$ respectively). The $\mathrm{LR}+$ values were moderate to high (4.15 and 14.6 respectively). The AUC showed a good diagnostic performance ( 0.80 and 0.71 respectively). On the other hand, the history of previous infection and history of antischistosomal treatment showed a low sensitivity (27.0\% and $21.4 \%$ respectively), low PPV (37.8\% and $17.5 \%$ respectively), low $\mathrm{LR}+$ ( 2.75 and 0.96 respectively) and the AUC indicated poor diagnostic performance ( 0.59 and 0.50 respectively).

Dipstick testing and visual inspection of urine samples showed that microhaematuria only, both micro- plus macrohaematuria and all microhaematuria had moderate to high sensitivity values $(61.9 \%, 31.0 \%$ and $92.9 \%$ respectively), high specificity $(94.0 \%, 100 \%$ and $94.0 \%$ respectively), high PPV (69.6\%, 100\% and $77.5 \%$ respectively), high NPV (91.8\%, 86.8\% and 98.3\% respectively) with good, sufficient and excellent AUC values $(0.78,0.66$ and 0.93 respectively). 


\begin{tabular}{|c|c|c|c|c|c|c|c|c|}
\hline \multirow[t]{3}{*}{ Age (years) } & \multicolumn{4}{|c|}{ Reporting haematuria } & \multicolumn{4}{|c|}{ Total } \\
\hline & \multicolumn{2}{|c|}{$\begin{array}{c}\text { Males } \\
(n=47)\end{array}$} & \multicolumn{2}{|c|}{$\begin{array}{c}\text { Females } \\
(n=29)\end{array}$} & \multicolumn{2}{|c|}{$\begin{array}{c}\text { Males } \\
(n=305)\end{array}$} & \multicolumn{2}{|c|}{$\begin{array}{l}\text { Females } \\
(n=391)\end{array}$} \\
\hline & No. & $\%$ & No. & $\%$ & No. & $\%$ & No. & $\%$ \\
\hline$<12$ & 3 & 6.4 & 1 & 3.5 & 37 & 12.1 & 51 & 13.0 \\
\hline $12-<13$ & 17 & 36.2 & 13 & 44.8 & 96 & 31.5 & 187 & 47.8 \\
\hline $13-<14$ & 15 & 31.9 & 9 & 31.0 & 103 & 33.8 & 118 & 30.2 \\
\hline $14-16$ & 12 & 25.5 & 6 & 20.7 & 69 & 22.6 & 35 & 9.0 \\
\hline
\end{tabular}

\section{Discussion}

It well known that haematuria, dysuria and proteinuria are the common symptoms and signs of urinary schistosomiasis. The present findings revealed that $78.6 \%$ of infected schoolchildren reported having dysuria compared with only $18.9 \%$ of those who were not infected. The difference was statistically significant. The infection among most children was of low intensity and this could explain the absence of dysuria among about one-fifth of infected schoolchildren. The sensitivity and specificity of self-reported dysuria compared with microscopic examination of urine in our study $(78.6 \%$ and $81.1 \%$ respectively) were higher than values obtained by some researchers in Cameroon (52\% and 65\% respectively) [6] and in Nigeria (25\% and $87.5 \%$ respectively) [7]. In addition, the moderate PPV (47.8\%), high NPV (94.5\%), moderate LR+ (4.15) and AUC of 0.8 indicated a good diagnostic performance for the item on history of self-reported dysuria using the morbidity questionnaire.

The results also revealed that $46.0 \%$ of infected schoolchildren reported having haematuria compared with $3.2 \%$ among those who were not infected, with a statistically significant difference. Most of those with selfreported haematuria (72.2\%) had high intensity of infection, compared with $41.7 \%$ who were not infected, with a statistically significant difference. These findings are similar to those of other investigators [2], who reported an association between self-reported haematuria, prevalence of infection and level of intensity determined by egg count. In the current study, the sensitivity and specificity of self-reported haematuria were $46.0 \%$ and $96.8 \%$ respectively. The sensitivity is close to that reported by Mafe (44\%) [8], but lower than that obtained by Ba'amer (74\%) [2]. The specificity was higher than that obtained by these 2 previous authors ( $81 \%$ and $89 \%$ respectively). Therefore the item on history of selfreported haematuria revealed a good diagnostic performance. This finding was in accordance with that reported by other investigators [9], who emphasized that the questionnaire approach could be adopted for the diagnosis of $S$. haematobium and that morbidity indicators were useful for individual infection status.

Two studies carried out in Tanzania observed that girls were more likely than boys to be missed in self-diagnosis screening for S. haematobium $[10,11]$. These studies confirmed previous reports of under-reporting of haematuria and schistosomiasis by girls at school level. As a result, the sensitivity and specificity of the questionnaire may differ by sex just as it may differ by age and overall endemicity [12]. They suggested that the onset of menses influences girls' willingness to report haematuria. This trend was also noticed in the present study, as about $51.7 \%$ of the girls who reported haematuria were within the age group of 13-16 years, which coincides with the mean age of menarche in Yemen, 14.4 years [13].

Our findings revealed that $27.0 \%$ of children who were infected reported having a history of previous infection compared with $9.8 \%$ who were not infected, and that $27.8 \%$ had a high intensity of infection. These findings were in agreement with those reported by other authors [14]. The proportion of children with a history of Schistosoma infection and who were still infected could be attributed to the fact that children were not receiving the correct dose of praziquantel or had refused treatment because of fear of its side-effects, especially abdominal discomfort, diarrhoea, dizziness and sleepiness [15]. The diagnostic performance of the reported history of previous infection was characterized by low sensitivity, PPV, LR + and poor AUC values $(27.0 \%, 37.8 \%, 2.75$ and 0.59 respectively) showing a poor diagnostic performance of this item in the morbidity questionnaire.

About $21 \%$ of infected children had a history of previous antischistosomal treatment 1 month prior the implementation of the study and 33.3\% of them had high intensity of infection. This finding could be attributed to several factors, such as incomplete administration of the specified dose of praziquantel, exposure to new infection or the possibility of drug resistance. Other researchers recorded that the failure rate of praziquantel was $50 \%, 18.5 \%$ and $12.5 \%$ among preschool, schoolchildren and adults respectively after the third treatment regimen in 3 villages in Lower Egypt 
[16]. The diagnostic performance of self-reported previous antischistosomal treatment as a screening tool was poor in our study, due to low sensitivity, PPV, LR+ and poor value of AUC (21.4\%, $17.5 \%$, 0.96 and 0.50 respectively)

Microhaematuria is considered a more sensitive and specific screening test for S. haematobium than is proteinuria [17]. Reagent strips are capable of detecting minute amounts of blood in urine, as low as only 5 erythrocytes per $\mathrm{mL}$, equivalent to $0.015 \mathrm{mg}$ of soluble haemoglobin per $100 \mathrm{~mL}$ of urine [18]. A previous study reported that a higher proportion of individuals with $>50$ eggs per $10 \mathrm{~mL}$ urine could be detected using a single reagent strip examination for microhaematuria than by a single urine filtration test [19].

This school-based survey was performed mainly to assess the reliability of microhaematuria alone as an indirect indicator for the presence of urinary schistosomiasis compared with the goldstandard method. The results showed a significant relationship between microhaematuria and the prevalence and intensity of infection among the studied schoolchildren. Of those who were positive by microscopy, $61.9 \%$ were also positive for microhaematuria by reagent strips, a relatively high sensitivity of $61.9 \%$ and high specificity of $94.0 \%$. A previous study reported a higher sensitivity value than our results (89.6\%) [20]. The observed variations in the sensitivity and specificity values of microhaematuria alone could be due to regional differences of prevalence and the intensity of infection of urinary schistosomiasis as well as the varying quality of the reagent strips from different producers. Nevertheless, our findings revealed a relatively high PPV (69.6\%), which means that $30.4 \%$ of children probably did not have schistosomiasis in the presence of microhaematuria. The high NPV indicated that the probability of not having schistosomiasis among children without microhaematuria was $91.8 \%$. Therefore $8.2 \%$ of children had the probability of having the disease in the absence of microhaematuria. The LR+ was 10.4, which indicates that the children who had microhaematuria were about 10 times more likely to have Schistosoma infection than those who had no microhaematuria. Besides, the AUC showed a good diagnostic value (0.78). These diagnostic accuracy tests indicated a good diagnostic performance. Our findings were in accordance with those reported in Abyan and Taiz Governorates, Yemen [2].

The current results revealed that $31.0 \%$ of infected schoolchildren had both micro- and macrohaematuria and all of them (100\%) had a high intensity of infection. There was a significant relationship between microhaematuria with concomitant macrohaematuria and the prevalence and intensity of S. haematobium infection.

The diagnostic performance of microhaematuria plus macrohaematuria by using reagent strips and visual inspection of urine samples recorded a low sensitivity (31.0\%), which could be due to several reasons. First, the present study was limited since only one urine specimen was collected. Secondly, instructions were given to children to conduct physical exercise prior to urine collection but 
most of the children did not respond, especially the girls. A previous study has shown that repeated examination of urine specimens over consecutive days and exercise prior to urine collection improved egg detection [21]. Finally, children who either passed blood in urine or tested positive for S. haematobium infection might be referred to health facilities for treatment and hence decrease the accuracy of sensitivity. The high values of specificity, PPV, NPV, LR+ and sufficient AUC (100\%, 100\%, $86.8 \%, 355$ and 0.66 respectively) indicated a good diagnostic performance. The same findings were also recorded by several authors $[2,8]$.

The diagnostic performance of all microhaematuria in the present study revealed a high sensitivity (92.9\%) and high specificity (94.0\%). The PPV was $77.5 \%$ and the high NPV (98.3\%) showed that the probability of children having the disease in the absence of such microhaematuria was only $1.7 \%$. The LR+ (15.6) indicated that children who had microhaematuria were about 16 times more likely to have Schistosoma infection than those who did not have all microhaematuria. The AUC showed an excellent diagnostic value (0.93). These diagnostic accuracy tests revealed a good diagnostic performance of microhaematuria as an indicator for the detection of urinary schistosomiasis infection.

The urine reagent strip method is a cheap (at least 3 times cheaper than the filtration diagnostic method [22]), easy to perform and rapid assessment method for identifying infected individuals and communities at risk of $S$. haematobium infection. It can be used in the primary health-care settings for screening and monitoring $S$. haematobium infection [23]. In this study we demonstrate that the use of urine reagent strips coupled with simple questionnaire might be considered for the diagnosis of $S$. haematobium where microscopy is unavailable

\section{Acknowledgements}

The authors wish to thank all the schoolchildren of Battis village who participated in the study and we are very grateful to their families. We wish to express our gratitude to the administrative officials of the school who were very cooperative during the execution of the study. Thanks are also extended to the local health authorities and those who work in the laboratories for supplying us all the needed equipment and materials for field study and for supplying the required praziquantel to treat the infected children.

Funding: This investigation did not receive any financial support from any authorities.

Competing interests: None declared.

\section{References}

1. Oshish A et al. Towards nationwide control of schistosomiasis in Yemen: a pilot project to expand treatment to the whole community. Transactions of the Royal Society of Tropical Medicine and Hygiene, 2011, 105:617-627.

2. Ba'amer AA. Two practical and cost effective methods for urinary schistosomiasis screening in Yemeni schoolchildren. Iranian Journal of Public Health, 2009, 38:78-83.

3. Lucas AO, Gilles HM, eds. A new short textbook of preventive medicine for the tropics, 3rd ed. London, Hodder Arnold, 1990.

4. Richards FO Jr et al. An evaluation of quantitative techniques for Schistosoma haematobium eggs in urine preserved with carbolfuchsin. American Journal of Tropical Medicine and Hygiene, 1984, 33:857-861.

5. Simundic AM. Measures of diagnostic accuracy: basic definition. Journal of the International Federation of Clinical Chemistry and Laboratory Medicine, 2002, 19:2-8.

6. Takougang I et al. Hematuria and dysuria in the self-diagnosis of urinary schistosomiasis among school-children in Northern Cameroon. African Journal of Health Sciences, 2004, 11:121-127.

7. Fatiregun AA, Osungbade KO, Olumide EA. Diagnostic performance of screening methods for urinary schistosomiasis in a school-based control programme, in Ibadan, Nigeria. Journal of Community Medicine and Primary Health Care, 2005, 17:24-27.

8. Mafe MA. The diagnostic potential of three indirect tests for urinary schistosomiasis in Nigeria. Acta Tropica, 1997, 68:277-284.

9. El-Khoby T et al. The epidemiology of schistosomiasis in Egypt: summary findings in nine governorates. American Journal of Tropical Medicine and Hygiene, 2000, 62(Suppl.):88-99.
10. Guyatt $\mathrm{H}$ et al. The performance of school-based questionnaires of reported blood in urine in diagnosing Schistosoma haematobium infection: patterns by age and sex. Tropical Medicine and International Health, 1999, 4:751-757.

11. Ansell J et al. The effects of sex and age of responders on the reliability of self-diagnosed infection: a study of self-reported urinary schistosomiasis in Tanzanian school children. Social Science and Medicine, 2001, 53:957-967.

12. Kihara J et al. Preparing for national school-based deworming in Kenya: the validation and large-scale distribution of school questionnaires with urinary schistosomiasis. Tropical Medicine and International Health, 2011, 16:1326-1333.

13. Thomas F et al. International variability of ages at menarche and menopause: patterns and main determinants. Human Biology, 2001, 73:271-290.

14. Lengeler C, Utzinger J, Tanner M. Questionnaires for rapid screening of schistosomiasis in sub-Saharan Africa. Bulletin of the World Health Organization, 2002, 80:235-242.

15. The control of schistosomiasis. Geneva, World Health Organization, 1985 (Technical Report Series, No. 728).

16. Ismail MM et al. Treatment of schistosomiasis with praziquantel among school children. Journal of the Egyptian Society of Parasitology, 1994, 24:487-494.

17. Eltoum IA et al. Evaluation of haematuria as an indirect screening test for schistosomiasis haematobium: a population-based study in the White Nile province, Sudan. Acta Tropica, 1992, 51:151-157.

18. Savioli L et al. Control of morbidity due to Schistosoma haematobium on Pemba Island: programme organization and management. TropicalMedicine and Parasitology, 1989, 40:189-194. 
19. Savioli L et al. Control of morbidity due to Schistosoma haematobium on Pemba Island: egg excretion and hematuria as indicators of infection. American Journal of Tropical Medicine and Hygiene, 1990, 43:289-295.

20. Houmsou RS et al. Urine colour as a rapid indicator of urine schistosomiasis in Katsina-Ala and Buruku LGAs of Benue State, Nigeria. International Journal of Tropical Medicine, 2009 , 6:282-290.

21. Doehring E, Feldmeier H, Daffalla AA. Day-to-day variation and circadian rhythm of egg excretion in urinary schistosomiasis in the Sudan. Annals of Tropical Medicine and Parasitology, 1983, 77:587-594.

22. Lengeler $\mathrm{C}$ et al. Community-based questionnaires and health statistics as tools for the cost-efficient identification of communities at risk of urinary schistosomiasis. International Journal of Epidemiology, 1991, 20:796-807.

23. Kotb $\mathrm{MM}$ et al. Evaluation of the effectiveness of dipstick haematuria and proteinuria in screening Schistosoma haematobium infection among school children in upper Egypt. Journal of the Egyptian Public Health Association, 1996, 71:353-367. 